Journal for ImmunoTherapy of Cancer

\title{
A new role for circulating $T$ follicular helper cells in humoral response to anti- PD-1 therapy
}

\author{
Santiago Sánchez-Alonso, ${ }^{1}$ Giulia Setti-Jerez, ${ }^{1}$ Montserrat Arroyo, \\ Tathiana Hernández, ${ }^{1} M^{\mathrm{a}}$ Inmaculada Martos, ${ }^{2}$ Jose Miguel Sánchez-Torres, ${ }^{3}$ \\ Ramon Colomer, ${ }^{3}$ Almudena R Ramiro, ${ }^{2}$ Arantzazu Alfranca (1) ${ }^{1}$
}

To cite: Sánchez-Alonso $\mathrm{S}$, Setti-Jerez G, Arroyo M, et al. A new role for circulating $T$ follicular helper cells in humoral response to anti-PD-1 therapy. Journal for ImmunoTherapy of Cancer 2020;8:e001187. doi:10.1136/jitc-2020-001187

- Additional material is published online only. To view, please visit the journal online (http://dx.doi.org/10.1136/jitc2020-001187).

Accepted 10 August 2020 (c) Author(s) (or their employer(s)) 2020. Re-use permitted under CC BY-NC. No commercial re-use. See rights and permissions. Published by BMJ.

${ }^{1}$ Immunology Department, Hospital Universitario de la Princesa. Instituto de Investigación Sanitaria del Hospital Universitario de La Princesa, Madrid, Spain

${ }^{2} B$ Lymphocyte Lab, Fundación Centro Nacional de Investigaciones Cardiovasculares Carlos III (CNIC), Madrid, Spain ${ }^{3}$ Medical Oncology Department, Hospital Universitario de la Princesa, Madrid, Spain

\section{Correspondence to} Dr Arantzazu Alfranca; mariaaranzazu.alfranca@salud. madrid.org

\section{ABSTRACT}

Background Lung cancer is one of the most frequent malignancies in humans and is a major cause of death. A number of therapies aimed at reinforcing antitumor immune response, including antiprogrammed cell death protein 1 (anti-PD-1) antibodies, are successfully used to treat several neoplasias as non-small cell lung cancer (NSCLC). However, host immune mechanisms that participate in response to anti-PD-1 therapy are not completely understood.

Methods We used a syngeneic immunocompetent mouse model of NSCLC to analyze host immune response to anti-PD-1 treatment in secondary lymphoid organs, peripheral blood and tumors, by flow cytometry, immunohistochemistry and quantitative real-time PCR (qRT-PCR). In addition, we also studied specific characteristics of selected immune subpopulations in ex vivo functional assays.

Results We show that anti-PD-1 therapy induces a population of circulating T follicular helper cells (cTfh) with enhanced B activation capacity, which participates in tumor response to treatment. Anti-PD-1 increases the number of tertiary lymphoid structures (TLS), which correlates with impaired tumor growth. Of note, TLS support cTfh-associated local antibody production, which participates in host immune response against tumor. Conclusion These findings unveil a novel mechanism of action for anti-PD-1 therapy and provide new targets for optimization of current therapies against lung cancer.

\section{BACKGROUND}

Lung cancer is the leading cause of death by cancer among men and women worldwide. Non-small cell lung cancer (NSCLC) accounts for up to $85 \%$ of all lung cancer cases that includes two main entities: adenocarcinoma and squamous cell carcinoma. Both are associated with a poor prognosis, with a 5-year survival rate of $<20 \%$ of patients. ${ }^{1}$

Adaptive antitumor immune response takes place in secondary lymphoid organs (SLOs), where lymphocytes, mainly cytotoxic CD8+ T cells, are primed to recognize tumor cells and then recruited to tumor microenvironment to exert their effector function as tumor infiltrating lymphocytes (TILs). ${ }^{2}$ Similarly, antibody production facilitated by CD4+ lymphocyte help also occurs in SLOs, within specialized structures called germinal centers (GC). ${ }^{2}$ Follicular helper T cells (Tfh) constitute a specialized subset of CD4+ T lymphocytes that localizes in close interaction with $\mathrm{B}$ cells in GCs, and collaborates in the generation of high-affinity antibody responses. Mature Tfh cells participate in somatic hypermutation processes and clonal selection of GC B cells. ${ }^{3}$ One hallmark of the adaptive immune system is the generation of immunological memory. Several studies support the notion that GC responses generate memory $\mathrm{Tfh}$ in addition to memory B cells. Recently, a new subset of CD4+ T lymphocytes with Tfhlike immunophenotype (CD4+CXCR5+programmed cell death protein 1 (PD-1)+CCR7 low) were identified in peripheral blood of human and mice. ${ }^{3}$ These circulating Tfh (cTfh) are associated with a memory-like function, since they can reactivate after dendritic cell re-encounter, home to GCs and produce more cytokines than their GC counterparts. ${ }^{4}$ Likewise, some authors suggest that cTfh could provide robust B cell help during secondary antigen exposure to induce strong antibody responses, ${ }^{5}$ and discriminate among functional subpopulations of $\mathrm{cTfh}$ with different $\mathrm{B}$ activation capacity based on their expression of markers such as CXCR3, ICOS, PD-1 and CCR7. ${ }^{4-6}$ However, neither the specific precursor of cTfh cells nor the potential role of $\mathrm{cTfh}$ in antitumor immune response have been defined yet.

Adaptive antitumor response occurs at SLOs and in certain organized structures in tumor sites, called tertiary lymphoid structures (TLS). ${ }^{7}$ TLS are ectopic organized lymphoid aggregates comprised of distinct T-cell and B-cell zones, which reflect lymphoid neogenesis occurring in peripheral tissues. 
TLS presence is associated with favorable prognosis in most solid malignancies including NSCLC, ${ }^{8}$ as they help local presentation of tumor antigens to $\mathrm{T}$ cells, together with activation and differentiation of $\mathrm{T}$ and $\mathrm{B}$ cells. ${ }^{9}$ Local production of proinflammatory cytokines recruits lymphoid tissue inducer (LTi) cells to the site of inflammation. These LTis interact with stromal cells present in tumor microenvironment, which resemble lymphoid tissue organizers in SLOs. This initial phase of priming leads to secretion of chemokines as CXCL12, CXCL13, CCL19 and CCL21. These factors promote recruitment of lymphoid cells to the site of inflammation and regulate their organization in T-cell and B-cell zones. ${ }^{1011}$

Tumors have developed different strategies to escape antitumor immune response. ${ }^{12}$ Among these mechanisms, the role of PD-1-ligand 1 (PD-L1) axis was studied in depth because of its inhibitory function on T lymphocytes. ${ }^{13} 14$ However, PD-1 ligation triggers inhibitory signals in T lymphocytes, and participates in positioning and differentiation of Tfh cells within SLOs. Hence, PD-1 interacts with PD-L1 expressed by bystander B cells in GCs, restraining PI3K signaling necessary for Tfh motility downstream CXCR5-CXCL13 interaction. Additionally, PD-1 limits CXCR3 expression to confine Tfh cell inside the GC. ${ }^{15}$ Nevertheless, the precise mechanisms implicated in regulation of $\mathrm{Tfh}$ positioning and differentiation have not been defined at the time of writing.

As a result of PD-1 role in restraining host antitumor immune response, multiple clinical agents have been developed that target this molecule to treat patients with cancer. Hence, two monoclonal antibodies targeting PD-1, that is, nivolumab and pembrolizumab, have shown outstanding results in clinical trials, ${ }^{16-18}$ gaining Food and Drug Administration (FDA) approval as first-line therapy in several cancers (https://www.fda.gov/).

In the present study, we addressed the role of cTfh in antibody-mediated antitumor immune response following anti-PD-1 therapy, using a syngeneic immunocompetent mouse model of NSCLC. Our results demonstrate that anti-PD-1 induces a cTfh subpopulation with enhanced B activation capacity, associated with tumor response to treatment. We also show that anti-PD-1 increases the number of TLS, which support cTfh-associated antibody production and correlate with reduced tumor growth. These findings reveal a novel role of PD-1 in host immune response against lung cancer, and uncover previously unidentified mechanisms of action of anti-PD-1 therapy.

\section{METHODS}

\section{Cell lines and lentiviral production}

Mouse lung squamous cell carcinoma KLN205 cell line was purchased from Sigma-Aldrich (USA) and cultured in Eagle's Minimum Essential Medium (EMEM) supplemented with $10 \%$ heat-inactivated fetal bovine serum (Fisher Scientific, USA), 1X penicillin-streptomycin solution (Biowest, France), 2 mM L-glutamine (Lonza,
Switzerland) and 1X non-essential amino acid solution (Lonza, Switzerland), at $37^{\circ} \mathrm{C}$ and $5 \% \mathrm{CO}_{2}$.

To generate KLN205-Luc cell line, KLN205 cells were transduced with pHRSIN-CS-Luc-WdINotI.str lentiviral vector ${ }^{19}$ encoding luciferase gene, to allow tumor monitoring in mice. Lentiviral particles were produced in HEK-293T cells (ATCC, USA) by calcium phosphate transient transfection method. After 48 hours supernatants were collected and concentrated by ultracentrifugation at $26000 \mathrm{rpm}$ for 2 hours at $4^{\circ} \mathrm{C}$.

\section{Mice and tumor model}

All mouse studies were performed using DBA/2JRj male mice aged 12 weeks (Janvier Labs, France). KLN205 tumor cells were implanted subcutaneously, and tumor growth was monitored by bioluminescence with an in vivo image system (Vivo Vision IVIS 200) (PerkinElmer, USA), after intraperitoneal injection of $1.25 \mathrm{mg}$ of D-luciferin per mouse. Bioluminiscence was quantified with Living Image software (PerkinElmer), and animals were homogeneously sorted into treatment groups. For anti-PD-1 (clone RMP1-14, Bio X Cell, USA) and isotype-matched (clone 2A3, Bio X Cell) antibody treatment, mice were injected with $200 \mu \mathrm{g}$ of antibody intraperitoneally each 3-4 days. Tumors were harvested after 14 days of treatment initiation unless otherwise indicated.

\section{Flow cytometry}

Samples were blocked with mouse FcR Blocking Reagent (MiltenyiBiotec, USA), and then stained with the indicated antibodies. The antibodies included APC/Cy7 anti-interferon (IFN) $\gamma$, APC/Cy7 anti-CD38, BV421 anti-CCR7, BV510 anti-CXCR3, PerCP/Cy5.5 antiCXCR5, biotinylated anti-CXCR5 from Biolegend (USA); AF488 anti-CD4, eFluor506 anti-IL17A, PE anti-IL21, PE-Cy7 anti-CD44, PE-Cy7 anti-FoxP3 from eBiosciences (USA); FITC anti-CD69, PE anti-CD25, PerCP anti-CD3e, PE-Cy7 anti-IL4 from BD Biosciences (USA); biotinylated anti-PD-1 from R\&D systems (USA) and streptavidin-APC from Jackson Immunoresearch (USA). When necessary, red blood cell lysis was performed using BD Pharm Lyse lysing buffer (BD Biosciences). For intracellular staining, CytoFix/Perm kit (BD Biosciences) or FoxP3/Transcription Factor Staining Buffer Set (eBiosciences) were used according to the manufacturer's instructions. Cytometric data were collected using an FACS Canto II (BD Biosciences) and analyzed using FlowJo software (BD Biosciences). Gating strategy is shown in online supplemental figure 4 .

\section{Cell cultures and isolation}

For in vitro assays, CD4+ T cells were isolated from either peripheral blood or spleens of mice by negative selection using EasySep Mouse CD4+ T cell Isolation Kit (StemCell Technologies, Canada). Then, CXCR5+PD-1+ Tfh cells were further purified from isolated CD4+ T cells using Streptavidin MicroBeads in an autoMACs Separator (MiltenyiBiotec). In parallel, naïve T cells were 
purified by negative selection from isolated $\mathrm{CD} 4+\mathrm{T}$ cells by adding biotinylated anti-CD25 (BD Biosciences, USA) to an EasySep Mouse Memory T cell Depletion Cocktail (StemCell Technologies, Canada).For T cell activation assay, $4 \times 10^{4}$ naïve $\mathrm{T}$ cells, cTfh and spleen Tfh from antiPD-1-treated and isotype-treated mice were stimulated with Phorbol 12-myristate 13-acetate (PMA) (20 ng/ $\mathrm{mL})$ (Merck, Germany) plus ionomycin $(1 \mu \mathrm{M})$ (Merck, Germany) for 5 hours. Brefeldin A (Merck, Germany) was added for the last 2 hours to allow intracellular cytokine accumulation prior to flow cytometry staining.

\section{Antibody detection by ELISA and immunofluorescence}

Serum levels of IgM were determined in anti-PD-1-treated and isotype-treated mice using the Mouse IgM ELISA Quantitation set (Bethyl, USA) following the manufacturer's indications. Autoantibodies were detected by incubating HEp-2 ANA Slides and Rat Liver/Kidney/Stomach Slides (Inova Diagnostics, USA) with sera from mice with different treatments, and AF488 antimouse IgG (Life Technologies, USA) was used as secondary antibody for detection.

\section{Immunohistochemical analysis}

Tumors and spleens from anti-PD-1-treated and isotypetreated mice were fixed in $4 \%$ paraformaldehyde and embedded in paraffin; $3 \mu \mathrm{m}$ sections were stained with H\&E (Casa Álvarez, Spain). Additionally, samples were stained with m-IgG kappa (IgGk) BP-HRP (Santa Cruz Biotechnology, USA), anti-PD-1 (R\&D systems), antiCD4, anti-CD20 (Abcam, USA) and anti-Ki67 (Invitrogen, USA) antibodies. For immunofluorescence staining, tissue sections were incubated with primary antibody overnight at $4^{\circ} \mathrm{C}$ and then with secondary Alexa Fluor 647 anti-IgG $(\mathrm{H}+\mathrm{L})$, Alexa Fluor 555 anti-IgG $(\mathrm{H}+\mathrm{L})$ antibody or Alexa Fluor 647 Streptavidin (Invitrogen) for $30 \mathrm{~min}$ at room temperature. Finally, sections were counterstained with 4',6-Diamidino-2-phenylindole (DAPI) (Invitrogen) and evaluated by confocal laser scanning microscopy (acquired with a TCS SP5 microscope and processed with LAS-AF software; Leica, Germany).

TLS were defined as areas of dense and localized lymphocyte infiltration inside tumors or in their invasive margins. PD-1 and Ki67 expression was expressed as the percentage of positive cells in the selected area (TLS in tumors or GC in spleens) using Image J software. We considered three organization degrees within TLS as follows: grade I for localized but non-structured lymphoid aggregates with diffuse borders, grade II for discrete follicle-like structures without apparent GCs and grade III for follicle-like structures which contain distinct GCs. IgGk expression was estimated as the percentage of TLS positive for IgGk staining. Tumor size (S) was calculated from formalin-fixed paraffin-embedded (FFPE) sections using the formula $S=(\Phi \times \mathrm{L} \times \mathrm{W})$, where $\mathrm{L}$ is the largest tumor diameter and $\mathrm{W}$ the perpendicular tumor diameter.

\section{Chemokine expression analysis}

Total RNA was isolated from tumor FFPE slices using a Maxwell RSC RNA FFPE Kit in a Maxwell RSC device (Promega, USA), following the manufacturer's instructions. RNA was reverse transcribed to cDNA using the GoScript Reverse Transcription System (Promega). Quantitative real-time PCR (qRT-PCR) was performed with SYBR Green (Promega) in a CFX384 (BioRad, USA) and a QuantStudio 5 (Applied Biosystems, USA) detection systems. Primers used for qRT-PCR are listed in online supplemental table 1. $\beta$-Actin mRNA levels were used for normalization.

\section{Statistics}

Statistical analysis was carried out with Prism 5 (GraphPad Software). Data are presented as mean \pm SD unless otherwise specified. For comparison between populations, unpaired two-tailed Student's t-test or analysis of variance analysis was performed, as appropriate. For correlation analyses, Spearman's coefficient $r$ or Pearson's $r$ were calculated. $\mathrm{P}$ values $<0.05$ were considered to be statistically significant.

\section{RESULTS \\ Anti-PD-1 treatment induces a population of circulating Tfh with enhanced helper capacity in peripheral blood}

To assess the effect of anti-PD-1 therapy on immune response against tumors in our model, we first analyzed the profile of immune cells from peripheral blood, SLOs and TILs obtained from tumor-bearing mice after antiPD-1 or isotype treatment. To this end, we inoculated DBA/2JRj mice subcutaneously with syngeneic NSCLC KLN205 cells. Mice treated with control antibody showed progressive tumor growth, while treatment with antiPD-1 clearly impaired tumor development (figure 1A). We analyzed the distribution of immune cell subpopulations in both groups, and detected a significant increase in cTfh (CD4+PD-1+CXCR5+ cells) in peripheral blood of mice treated with anti-PD-1 antibodies. This increase was not observed in SLOs, which had comparable Tfh levels in both anti-PD-1-treated and isotype-treated mice (figure 1B,C). We performed CD25 and FoxP3 staining of blood and spleen Tfh cells, finding a significant reduction in follicular regulatory $\mathrm{T}$ cells (Tfr)/Tfh proportion between anti-PD1-treated and isotype-treated mice in peripheral blood but not in spleen, thereby ruling out a parallel increase in Tfr induced by PD-1 treatment (figure $1 \mathrm{~F}$ ).

To further characterize this population, we analyzed the expression of CD38, CXCR3 and CCR7 surface molecules, critically involved in Tfh function. ${ }^{6}$ We found that CXCR3 was reduced in cTfh from anti-PD-1-treated mice, while CD38 was significantly increased and CCR7 expression was comparable in cTfh from both groups. Of note, the immunophenotype of spleen Tfh cells was similar between treatments (figure 1D,E). These data indicate that anti-PD-1 therapy induces an increase of activated 
A

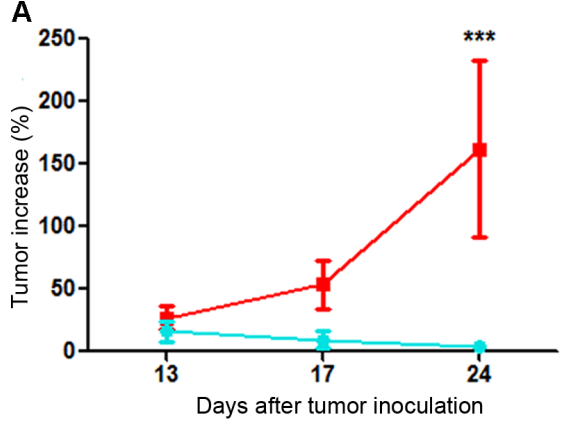

B

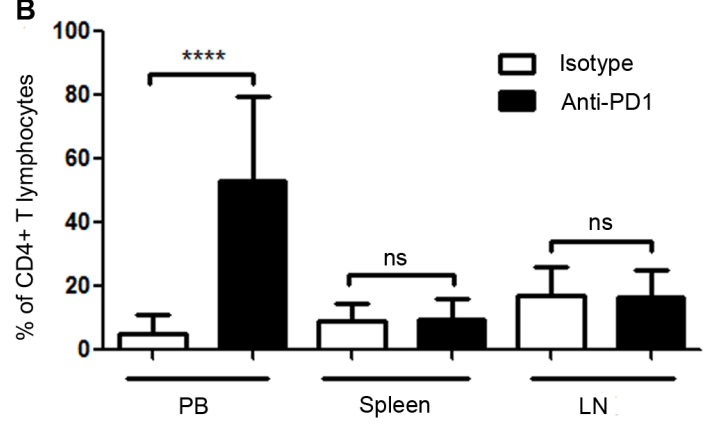

D
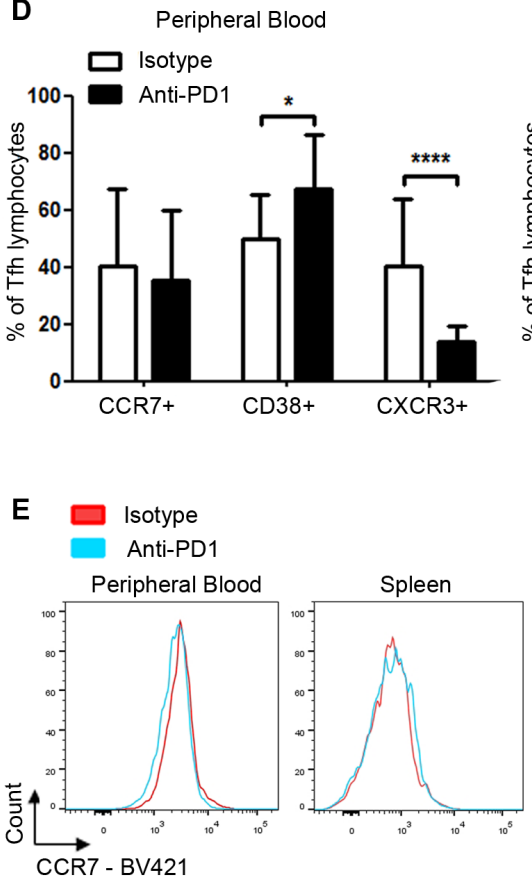

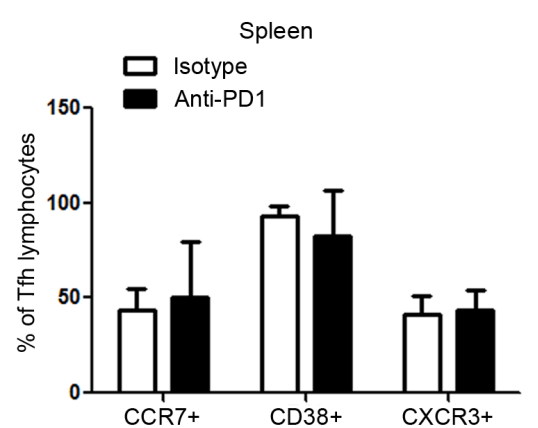

CCR7+

C Isotype

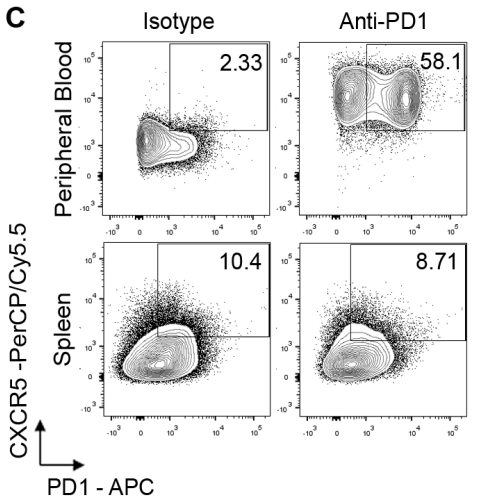

$\overrightarrow{\mathrm{PD} 1}$ - APC
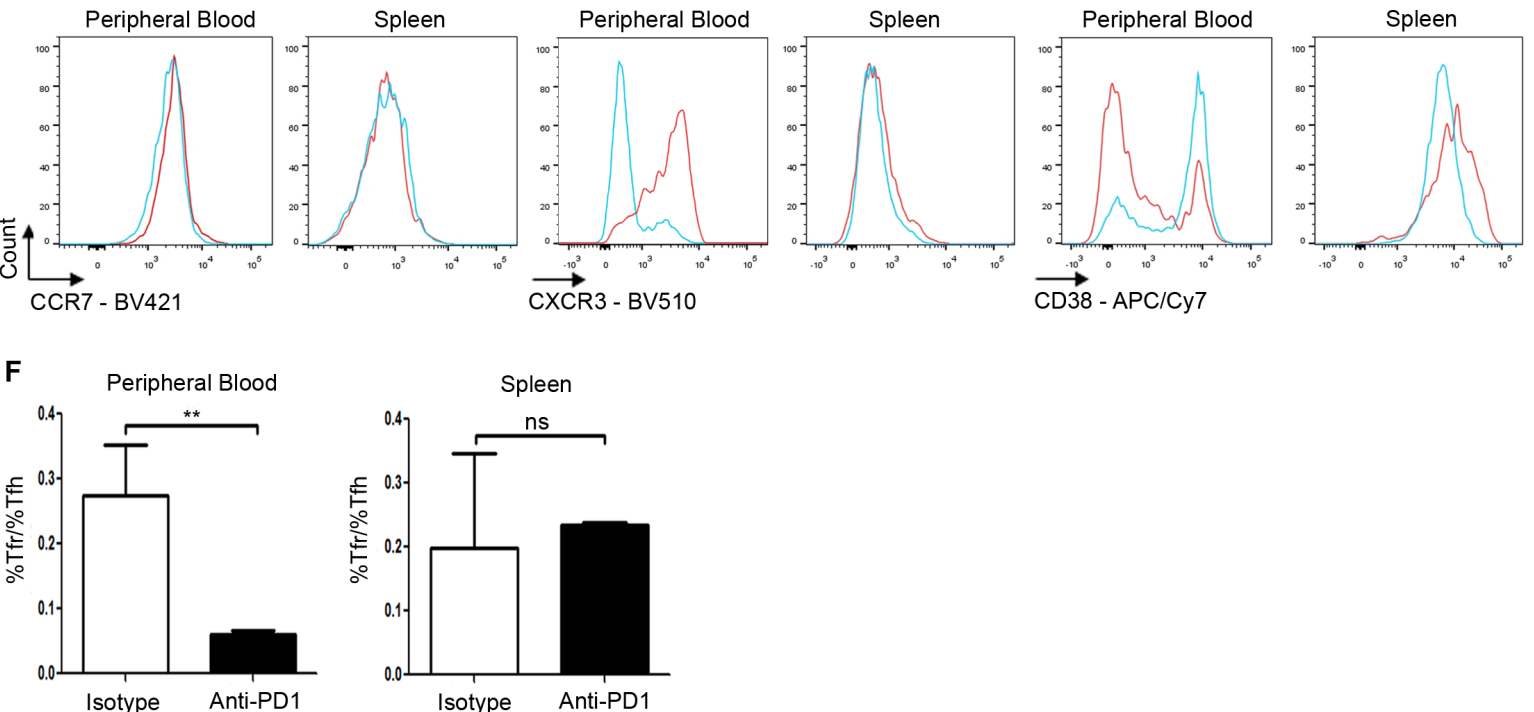

Figure 1 Antiprogrammed cell death protein 1 (anti-PD-1) treatment induces circulating T follicular helper cells (cTfh) in nonsmall cell lung cancer (NSCLC)-bearing mice. (A) Anti-PD-1 therapy reduces tumor development in KLN205 murine model of NSCLC. Graph represents tumor development in isotype-treated or anti-PD-1-treated mice $(n=9)$ estimated as percentage of tumor size at the onset of therapy, measured by bioluminescence. (B) Quantification of follicular helper T cells (Tfh) as percentage of CD4+ T cells in peripheral blood (isotype $n=29$ and anti-PD-1 $n=26$ ), spleen (isotype $n=18$ and anti-PD-1 n=15) and draining lymph nodes (isotype $n=8$ and anti-PD-1 $n=9$ ) from isotype-treated and anti-PD-1-treated mice. (C) Plots show representative CXCR5 and PD1 surface staining of Tfh lymphocytes from peripheral blood and spleen of anti-PD-1-treated and isotype-treated mice. Percentages of each subpopulation are indicated. (D) Percentage of CD38, CXCR3 and CCR7 expression in Tfh from peripheral blood and spleens of anti-PD-1-treated or isotype-treated mice; $n=9$ for peripheral blood and $n=12$ for spleen of isotype-treated mice, and $n=15$ for both peripheral blood and spleen of anti-PD-1-treated mice. (E) Representative histograms of surface markers showed in (D). (F) Follicular regulatory T cells (Tfr)/Tfh lymphocyte proportion in spleen (left) and peripheral blood (right) of anti-PD-1-treated and isotype-treated mice ( $n=8$ and $n=4$, respectively for peripheral blood and $n=3$ for spleen). Tfr and Tfh percentages (referred to CD4+ lymphocyte population, mean \pm SD) are the following: spleen, Tfr-isotype 0.49\% \pm 0.36\%; Tfr-anti-PD-1 1.13\% $\pm 0.53 \%$; Tfh-isotype 2.5\% $\pm 0.04 \%$; Tfh-anti-PD-1 4.82\% $\pm 2.19 \%$; peripheral blood, Tfrisotype $0.385 \% \pm 0.22 \%$; Tfr-anti-PD-1 3.82\% $\pm 1.19 \%$; Tfh-isotype $1.6 \% \pm 1.2 \%$; Tfh-anti-PD-1 $63.55 \% \pm 18.5 \%$. Tfr population was quantified as CD25+FoxP3+ cells within CD4+CXCR5+PD-1+ cells. ns, not significant. ${ }^{*} P<0.05$; ${ }^{* \star} p<0.01 ;{ }^{* * *} p<0.001$; ${ }^{* * * *} \mathrm{p}<0.0001$. 
cTfh in peripheral blood of treated mice, which display an immunophenotype compatible with a helper subpopulation. To gain insight into functional attributes of these cells, we measured cytokine production by $\mathrm{cTfh}$ and naïve CD4+ T cells from peripheral blood of anti-PD-1-treated and isotype-treated mice. Isolated cTfh were cultured in vitro with PMA/ionomycin (P/Io) for 5 hours, and intracellular staining of interleukin (IL)-21, IL-4, IL-17 and IFN $\gamma$ was performed. A selective induction of IL-21 and IL-4 was observed in cTfh from anti-PD-1-treated mice as compared with the isotype group (figure 2A). Conversely, both anti-PD1-treated and isotype-treated spleen Tfh showed a modest increase in IL-21 production on $\mathrm{P} / \mathrm{Io}$ treatment, with no variation in IL-4 level in any condition (figure 2A,B). Effective cell activation by $\mathrm{P} / \mathrm{Io}$ in all cases was confirmed through CD69 surface staining (figure 2C). We found no differences in IL-21 or IL-4 expression in $\mathrm{P} /$ Io-stimulated CD4+ T-naïve lymphocytes from any condition (figure 2D,E). Altogether, these data suggest that anti-PD-1 treatment specifically induces a circulating Tfh population with increased B cell activation capacity.

\section{Contribution of anti-PD-1 to antitumor antibody response}

To evaluate whether there was a parallel in vivo B cell activation response in anti-PD-1-treated mice, we first analyzed Ig levels in the serum of mice treated with either anti-PD-1 or isotype control antibodies. Tumor development induced a significant increment of serum IgM concentration, which was further increased by anti-PD-1 therapy (figure $3 \mathrm{~A}$ ).

To assess the effective production of antibodies directed against tumor cells, we carried out flow cytometry experiments with KLN205 cells incubated with sera from control and tumor-bearing mice. Unlike control animals, positive surface staining could be detected with sera from tumor-bearing mice (figure 3B). Parallel experiments with non-related B16 murine melanoma cells showed no staining in either condition (figure 3B). In addition, several autoimmune adverse effects were described in patients undergoing therapy with immune checkpoint inhibitors ${ }^{20}$; we, therefore, analyzed whether the increase of Ig level in anti-PD-1-treated mice was associated with the development of an autoimmune condition. Immunofluorescence assays revealed the appearance of autoantibodies in parallel to tumor development, whose titer slightly decreased with anti-PD-1 treatment (online supplemental figure 1), suggesting a paraneoplastic autoimmune syndrome, unrelated with anti-PD-1 therapy.

Altogether, these data show that tumor growth is associated with the development of an antitumor humoral response, which is further enhanced by anti-PD-1 treatment.

\section{Anti-PD-1 promotes germinal center reaction in spleen}

To assess the presence of GCs in SLOs, we analyzed expression and distribution of PD-1 and Ki67 markers by immunohistochemistry in spleens of tumor-free (control),
anti-PD-1-treated and isotype-treated mice. Tfh present in GCs express high levels of surface PD-1, and secrete ILs that promote proliferation, survival and differentiation of B lymphocytes. For this reason, we analyzed expression and distribution of PD-1 and Ki67 markers by immunohistochemistry in spleens of tumor-free (control), anti-PD-1treated and isotype-treated mice. We confirmed by CD4 staining that PD-1 expression in GCs corresponded to Tfh (online supplemental figures 2A and 4A). Similarly, we verified through CD20 staining that GC Ki67+ cells represented proliferating B lymphocytes (online supplemental figure 2A). We could detect a limited number of cells positive for either PD-1 or Ki67 staining in control mice (online supplemental figure 2B). However, tumor development was accompanied by the presence of both PD-1 and Ki67+ splenic GCs, which was further increased by anti-PD-1 treatment; likewise, the proportion of PD-1+ and Ki67+ cells inside GCs was significantly higher in anti-PD-1-treated mice (figure 4B,C). Notably, we could establish a significant correlation between PD-1 and Ki67 expression in GCs, probably reflecting Tfh-dependent B cell activation in these structures (figure 4D). Nevertheless, we did not find any significant relationship between expression of either PD-1 or Ki67 in splenic GCs and tumor response to anti-PD-1 treatment (figure 4E). These data support that anti-PD-1 therapy promotes in vivo B cell activation in spleen, which is not associated with tumor sensitivity to treatment.

\section{Anti-PD-1 therapy increases tumor TLS number and promotes in situ antibody generation}

Since we did not find any correlation between Tfhdependent B cell activation in spleen and tumor response to therapy, we evaluated the potential contribution of tumor ectopic lymphoid structures to antitumor immune response. We first quantified TLS in tumor samples from isotype-treated and anti-PD-1-treated mice, and observed a significant increase in TLS number in tumors from antiPD-1-treated mice, which displayed higher degree of organization than isotype-treated control group (figure 5A and online supplemental figure $3 \mathrm{~A}$ ), and inversely correlated with tumor size (figure 5A). A wide range of inflammatory and chemoattractant factors are involved in migration of immune cells to tumor microenvironment and regulation of different stages of TLS development. ${ }^{21}$ To study the implication of these factors in anti-PD-1induced TLS formation, we analyzed mRNA expression level of lymphotoxin (LT)- $\alpha$, LT- $\beta$, tumor necrosis factor (TNF- $\alpha$, CCL19, CCL21, CXCL12, CXCL13, CXCL9 and CXCL10 in tumor samples from anti-PD-1-treated and isotype-treated mice. Strikingly, CCL21 was the only chemokine with a significant increase in anti-PD-1-treated mice, while reduced expression of LT- $\alpha$ and LT- $\beta$, TNF- $\alpha$, CXCL12 and CXCL13 was detected in these mice. CCL19, CXCL9 and CXCL10 mRNA expression remained unaltered (figure 5B and online supplemental figure 3B). In addition, CCL21 increase secondary to anti-PD-1 treatment was directly related to both TLS presence and 
A

Peripheral Blood cTfh

Spleen Tfh
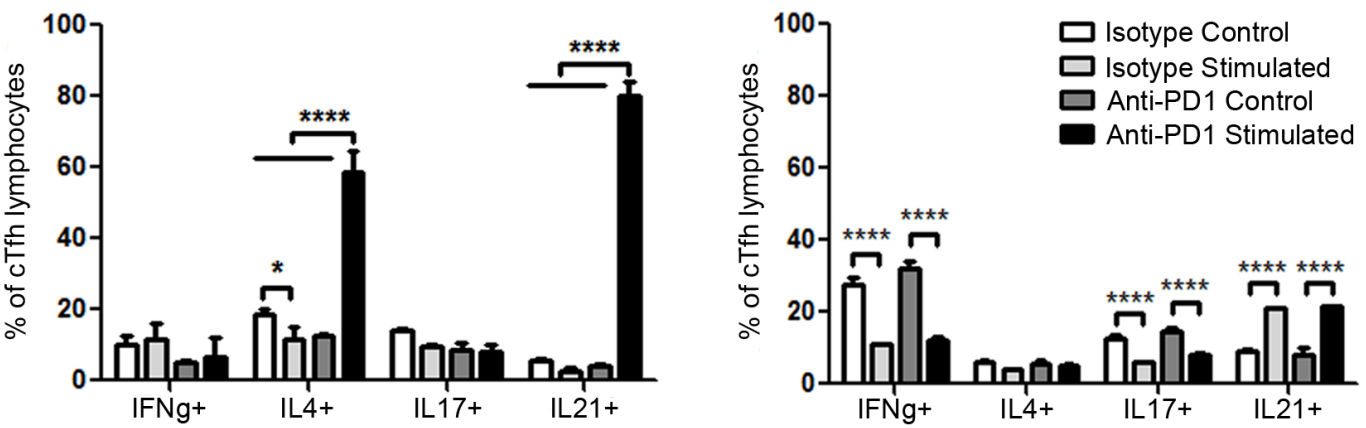

B
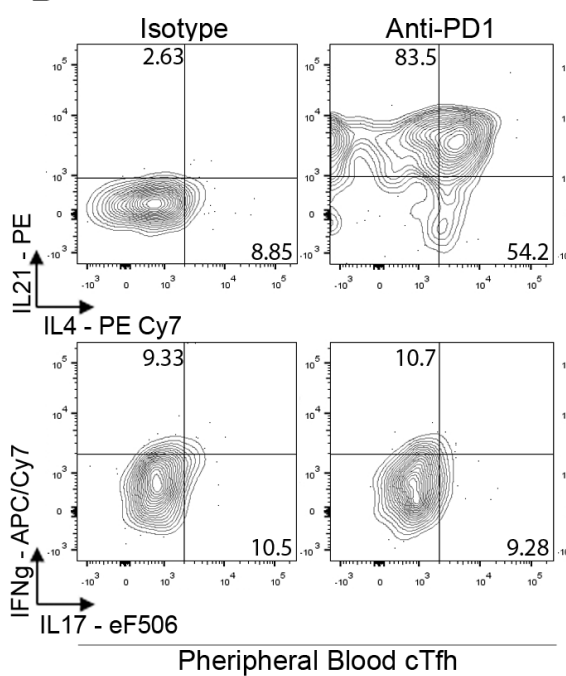

Pheripheral Blood cTfh

D

Peripheral Blood

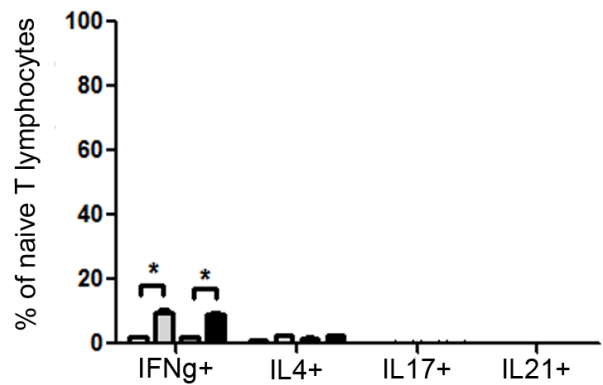

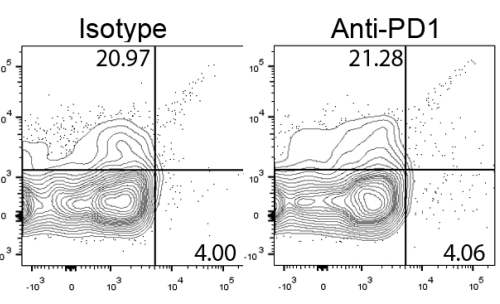

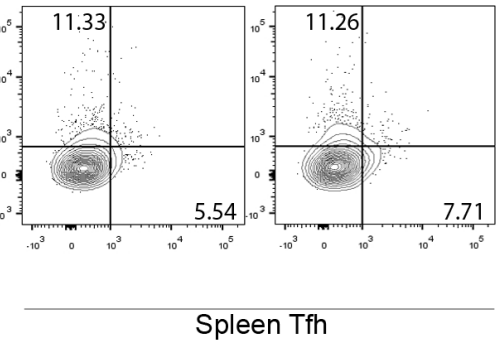

Spleen Tfh

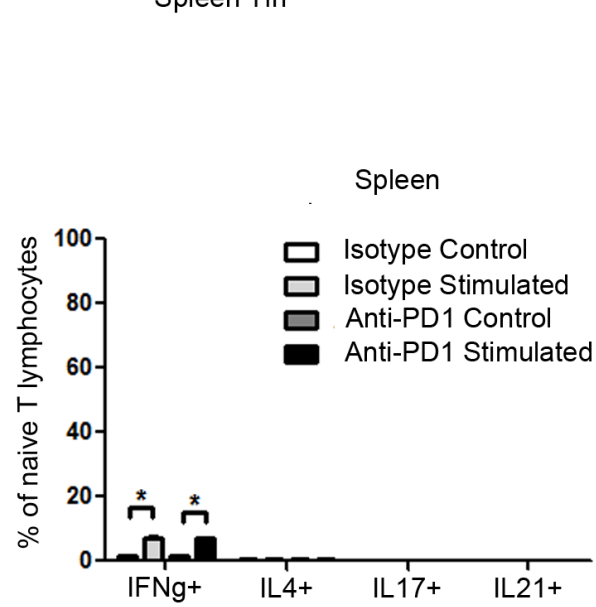

C
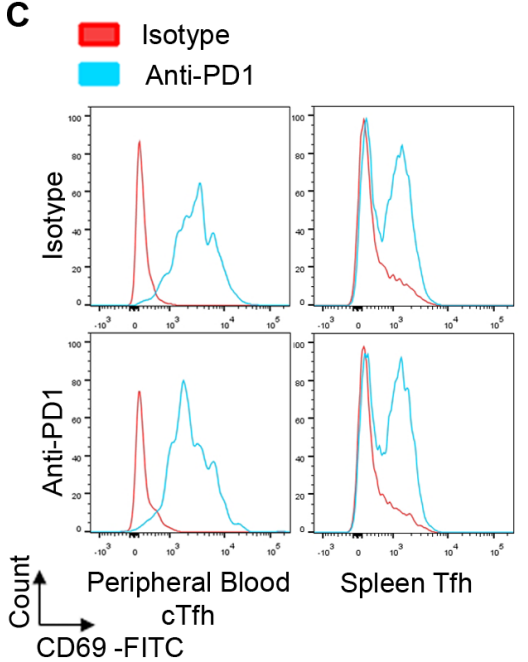

E

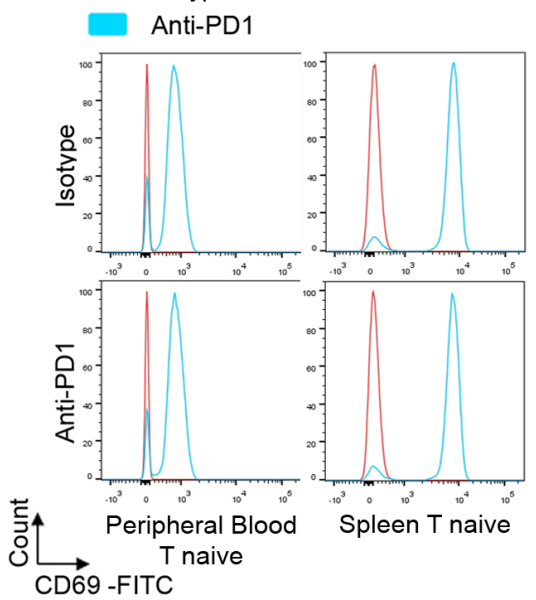

Figure 2 B cell activation capacity of follicular helper T cells (Tfh) from isotype-treated and antiprogrammed cell death protein 1 (anti-PD-1)-treated mice. (A) Quantification of interferon (IFN) $\gamma$, interleukin (IL)-4, IL-17 and IL-21 production in circulating T follicular helper cells (cTfh) and Tfh from spleen of anti-PD-1-treated and isotype-treated mice. For stimulation experiments, Tfh were isolated and pooled from isotype-treated and anti-PD-1-treated mice ( $n=7$ and $n=4$, respectively). They were subsequently treated for 5 hours with PMA/ionomycin (P/lo) plus brefeldin for the last 2 hours, and cytokine production was assessed by flow cytometry. (B) Representative plots of data showed in (A). Percentages of IFN $\gamma+$, IL-4+, IL-17+ and IL-21+ subpopulations are included. (C) Histograms show CD69 expression in Tfh from different conditions stimulated as in (A) to confirm effective cell activation. (D) Quantification of IFN $\gamma$, IL-4, IL-17 and IL-21 expression in P/lo-stimulated and control naïve CD4+ T lymphocytes from peripheral blood and spleen of anti-PD-1-treated and isotype-treated mice. Naïve T lymphocytes were isolated and pooled from seven isotype-treated mice and four anti-PD-1-treated mice, respectively. (E) Histograms show CD69 expression in T-naïve cells from different conditions stimulated as in (A) to confirm effective cell activation. ${ }^{*} \mathrm{P}<0.05 ;{ }^{* \star \star \star} \mathrm{p}<0.0001$. 
A

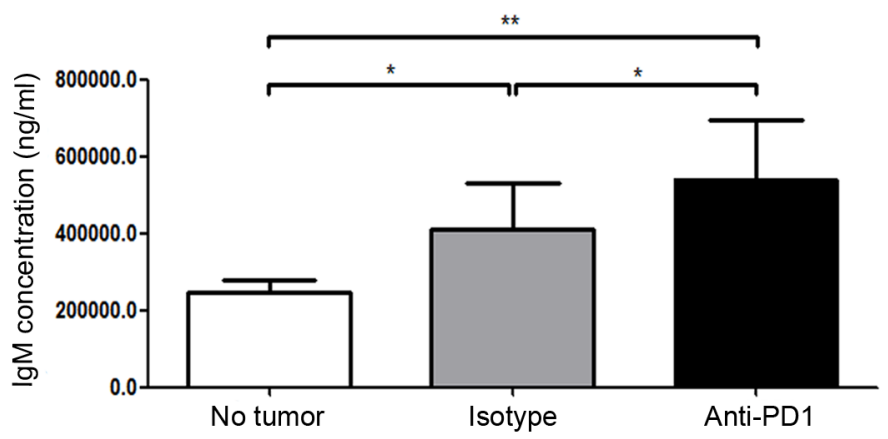

B

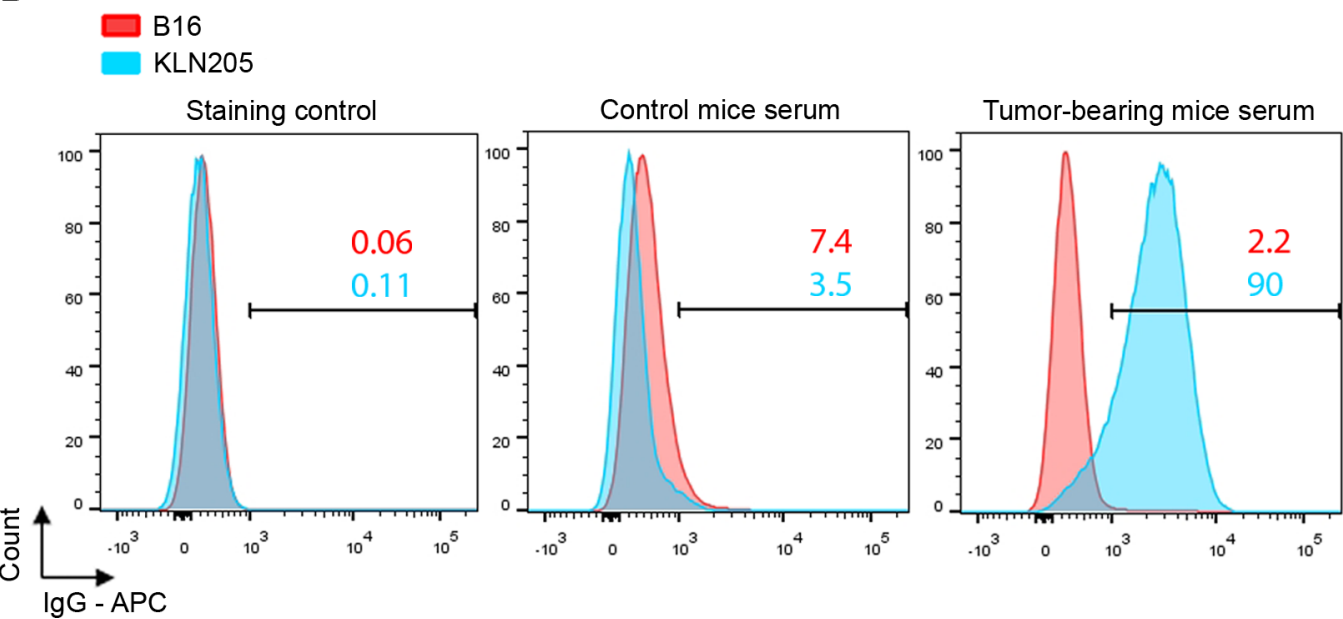

Figure 3 Antiprogrammed cell death protein 1 (anti-PD-1) therapy promotes an antibody-mediated immune response. (A) Quantification of serum IgM levels from healthy mice $(n=3)$, and anti-PD-1-treated $(n=14)$ and isotype-treated $(n=13)$ tumorbearing mice. (B) Flow cytometry staining of B16 (red) and KLN205 (blue) tumor cells with IgG from serum derived from healthy and tumor-bearing mice. Percentages of positive B16 and KLN205 cells are indicated. ${ }^{*} \mathrm{P}<0.05 ;{ }^{* *} \mathrm{P}<0.01$.

number (figure 5C). These results indicate that anti-PD-1 treatment increases the number of tumor TLS in parallel with a reduction in tumor burden, through a mechanism that may involve CCL21.

To analyze Tfh-mediated B cell activation in TLS, we examined PD-1 and Ki67 expression in these structures (figure 6A). We found a significant increase of positive cells for either marker in TLS from anti-PD-1-treated tumors, specifically in GC-like areas (figure 6B). Remarkably, anti-PD-1 responsive mice showed higher proportion of Ki67+ cells and a significant increment in PD-1+ cells in tumor TLS than non-responsive mice (figure 6C). Moreover, the increased proportion of PD-1+ and Ki67+ cells in TLS positively correlated with expansion of cTfh in peripheral blood from anti-PD-1-treated mice, whereas the percentage of spleen Tfh did not show any significant correlation with these markers (figure 6D). In the light of these findings, we sought to evaluate the potential contribution of TLS to the observed antibody response, so we examined mRNA expression level of soluble IgG1, IgG2b, IgG3 and IgM in tumor samples from anti-PD-1-treated and isotype-treated mice. We detected a strong expression of IgG1 together with lower levels of IgG2b, IgG3 and soluble IgM, which slightly increased in PD-1-treated mice (figure 7A). To identify the specific site of IgG production in these tissues, we performed IgGk) staining of tumor sections, and we could determine that TLS were indeed a prominent source of IgG (figure 7B). Moreover, we found a significant increase of IgGk-positive TLS in anti-PD-1-treated as compared with isotype-treated mice (figure 7B). Likewise, augmented proportion of IgGkproducing TLS correlated with both expansion of cTfh in anti-PD-1-treated mice and increased presence of PD-1+ GCs in tumor TLS (figure 7C).

Altogether, these data suggest that anti-PD-1 treatment induces B cell activation and increased antibody production within tumor TLS, associated with cTfh increase in peripheral blood.

\section{DISCUSSION}

Immunotherapy with checkpoint inhibitors has shown to be effective in a relevant percentage of patients with lung cancer, leading to increased survival rates. ${ }^{16-18}$ Here, we describe a novel mechanism of action of anti-PD-1 antibodies using a mouse squamous lung cancer model, which unveils a previously unknown role for PD-1 in the immune response against these tumors. Hence, anti-PD-1 promotes a significant increment in activated $\mathrm{cTfh}$ with increased B activation capacity. In parallel, this treatment 
A
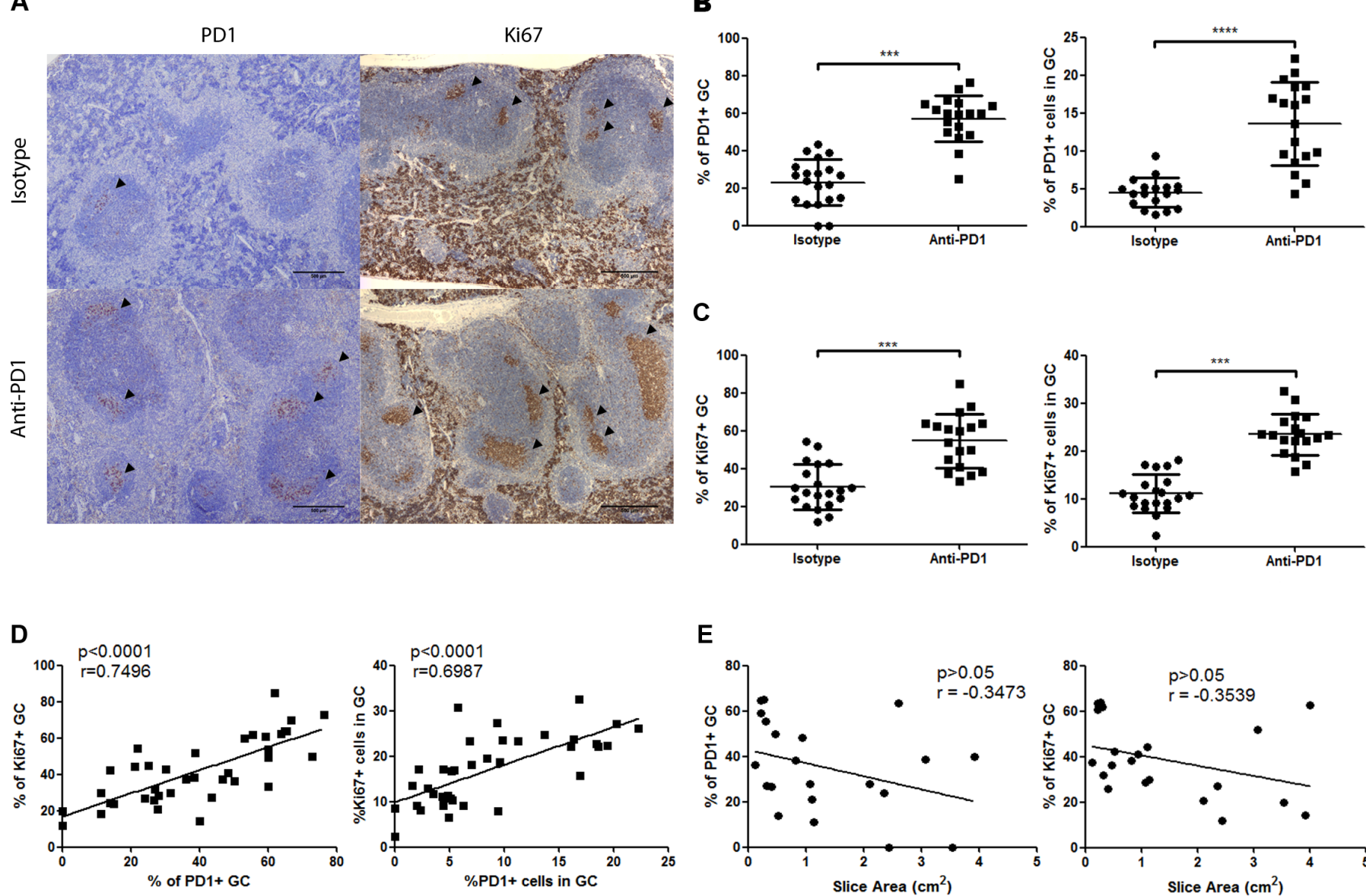

C
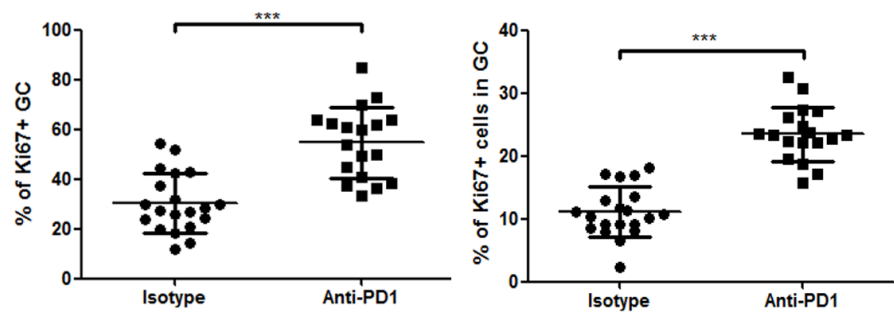

Figure 4 Antiprogrammed cell death protein 1 (anti-PD-1) treatment induces germinal centers (GC) reaction in spleen. (A) Immunohistochemical images of spleen sections from isotype-treated or anti-PD-1-treated mice. Sections were stained for PD-1 and Ki67. Arrowheads indicate positive staining in GCs for PD-1 (left panels) and Ki67 (right panels). Scale bars represent $500 \mu \mathrm{m}$. (B) Left, percentage of PD-1+ GCs in spleen sections of anti-PD-1-treated and isotype-treated mice; right, average percentage of PD-1+ cells in GCs present in spleen sections from anti-PD-1-treated and isotype-treated mice $(n=20$ and $n=18$, respectively). (C) Left, percentage of Ki67 GCs in spleen sections of anti-PD-1-treated and isotype-treated mice; right, average percentage of Ki67+ cells in GCs present in spleen sections from anti-PD-1-treated and isotype-treated mice $(n=20$ and $n=18$, respectively). (D) Correlation between either percentage of Ki67 and PD-1+ GCs (left) or average Ki67 and PD-1 cells inside GCs (right) ( $n=38$ ). Pearson's $r$ is shown for each correlation. (E) Correlation between tumor size and either percentage of PD-1+ GCs (left) or percentage of Ki67+ GCs (right) $(n=21)$. Pearson's $r$ is shown for each correlation. ${ }^{\star \star *} P<0.001 ;{ }^{* \star \star} p<0.0001$.

induces CCL21 production in tumors, together with an increment in TLS number related to impaired tumor growth. Of note, cTfh percentage correlates with the presence of PD-1-expressing and Ki67-positive proliferating cells specifically in TLS, which is associated with the production of antibodies able to recognize tumor cells and may collaborate in antitumor response (online supplemental figure 5).

We used KLN205 murine lung squamous carcinoma cell line as a model for in vivo experiments. ${ }^{22}$ However, human NSCLC are more frequently adenocarcinomas $(40 \%)^{23}$; therefore, despite anti-PD-1-mediated increase in cTfh probably relies on tumor type-independent mechanisms (see below in discussion), it will be of interest to confirm that our results are not restricted to squamous carcinoma by performing further experiments with additional NSCLC histological subtypes.
Immunohistochemistry experiments show an increment of PD-1-expressing cells in spleen GCs from mice treated with anti-PD-1 antibody (figure 4B) that correlates with that of Ki67 proliferation marker (figure 4C,D). Nevertheless, we did not detect a parallel Tfh increase in anti-PD-1-treated spleens by flow cytometry (figure 1B), which may be explained by $\mathrm{Tfh}$ redistribution towards GCs on treatment. In this regard, PD-1 plays a dual role in Tfh positioning in the follicle: PD-1 inhibits Tfh recruitment into GCs through its interaction with PD-L1 expressed by bystander B cells; and PD-1 promotes GC confinement of Tfh expressing high PD-1 levels through CXCR3 downregulation. ${ }^{15}$ Anti-PD-1 might also promote differentiation of naïve $\mathrm{CD} 4+$ cells into $\mathrm{Tfh}$, leading to an overall increase in Tfh number and contributing to the observed cTfh increment. Accordingly, previous reports have shown an expansion of $\mathrm{Tfh}$ population in 
A
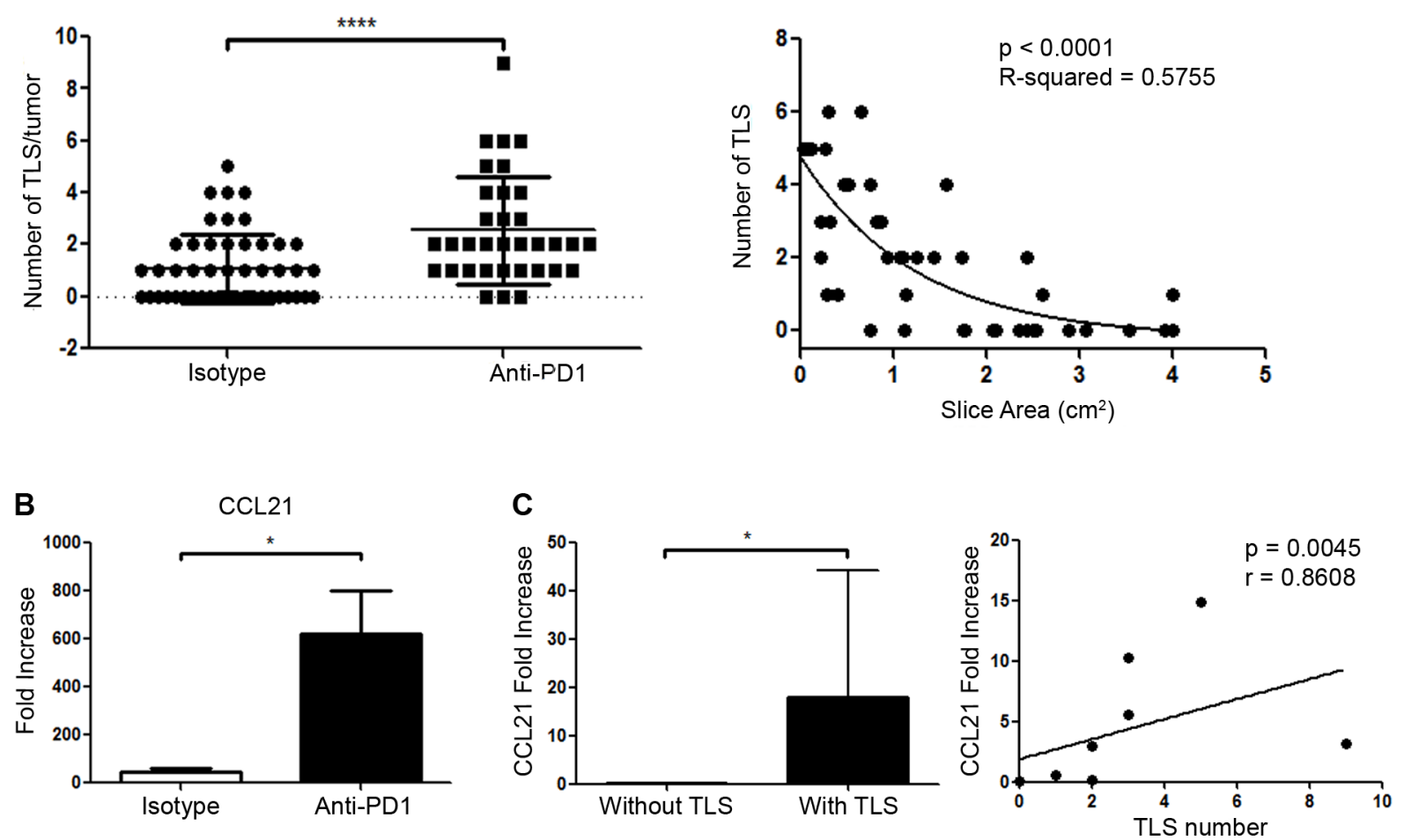

Figure 5 Tertiary lymphoid structures (TLS), number increases with antiprogrammed cell death protein 1 (anti-PD-1) therapy. (A) Left panel, quantification of TLS number per tumor from isotype-treated or anti-PD-1-treated mice ( $n=50$ and $n=34$, respectively); right panel, correlation between TLS number and tumor size $(n=46)$. R-squared is shown as a goodness-of-fit measure. (B) Quantification of expression of CCL21 chemokine, measured by quatitative real-time PCR (qRT-PCR) in whole tumor sections from isotype-treated and anti-PD-1-treated mice $(n=7$ and $n=6$, respectively). (C) Left panel, quantification of expression of CCL21 by qRT-PCR in anti-PD-1-treated tumor sections with or without presence of TLS ( $n=11)$. Right panel, correlation between level of expression of CCL21 and TLS number in anti-PD-1-treated tumors $(n=9)$. Spearman's coefficient $r$ is shown. CCL21 expression in anti-PD-1-treated tumors was normalized to average CCL21 expression level in isotype-treated tumors. ${ }^{*} \mathrm{P}<0.05 ;{ }^{* * * *} \mathrm{p}<0.0001$.

PD-1 knockout mice ${ }^{152425}$; also, overexpression of PD-1 in CD4+ $\mathrm{T}$ cells reduces $\mathrm{Tfh}$ presence in SLOs after immunization. ${ }^{15}$ Some authors have described mouse Tfh-like populations that egress from SLOs prior to GC reaction in a SAP-independent manner. ${ }^{4}$ These cTfh include a CCR $7{ }^{\mathrm{lo}} \mathrm{PD}-1^{\mathrm{hi}} \mathrm{CXCR} 5+\mathrm{CD} 4+$ subset with partial effector phenotype, which clearly resembles that of the cTfh population induced by anti-PD-1 in our experiments (figure $1 \mathrm{D}, \mathrm{E}$ ). CCR $7^{\mathrm{lo}} \mathrm{PD}-1^{\mathrm{hi}} \mathrm{cTfh}$ correlate with active Tfh differentiation, and are characterized by both a strong helper activity and the ability to further migrate into SLOs where they support GC reaction. ${ }^{4}$ In humans, influenza vaccination transiently induces a CD38+ICOS+ cTfh population that includes antigen-specific cells. These antigen-specific cells are highly activated and synthesize increased amounts of IL-21. ${ }^{26}$ Anti-PD-1 treatment induces a CD38+ cTfh population (figure 1D) with increased IL-21 production (figure 2A), which is consistent with an activated phenotype. Likewise, effector capacity of human cTfh associates with the expression of specific surface molecules. ${ }^{27}$ Thus, CXCR3- cells, which include both Tfh2 (CCR6-) and Tfh17 (CCR6+) subsets, correspond to cTfh with increased effector properties. CCR $7^{\text {lo }}$ PD- $1^{\text {hi }}$ phenotype associates with high ICOS expression and defines a population with the highest helper potential within each subtype. Our findings show that anti-PD-1-induced $\mathrm{CCR} 7^{\mathrm{lo}} \mathrm{PD}-1^{\text {hi }} \mathrm{cTfh}$ population has a reduced CXCR3 expression compared with cTfh in control mice (figure 1D), which is probably related to enhanced B cell activation capacity. Accordingly, these cells produce high levels of Tfh2 cytokines on stimulation with $\mathrm{P} / \mathrm{Io}$ (figure $2 \mathrm{~A}$ ). Of note, this population correlates with the presence of both PD-1-expressing and Ki67+ proliferating cells in TLS (figure 6D), which strongly suggests a role for cTfh on B cell activation in these structures. In the same line of thinking, combining treatment with anti-CTLA-4 and anti-PD-1 promotes Tfh infiltration in tumors with a parallel increment in IL-21 production, through a potential direct effect of these antibodies on Tfh cells. Moreover, tumor response to this therapy in animal models is highly dependent on CD4+ cells and Ig production by $\mathrm{B}$ cells. ${ }^{28}$ Similarly, recent reports indicate that the presence of B cell-rich TLS is a strong indicator of response to anti-PD-1 treatment in patients with melanoma, renal cell carcinoma and softtissue sarcomas. ${ }^{29-31}$ Furthermore, anti-CTLA-4 therapy in humans and in a murine melanoma model induces a CD4+ PD- $1^{\text {hi }}$ population both in peripheral blood and 
A

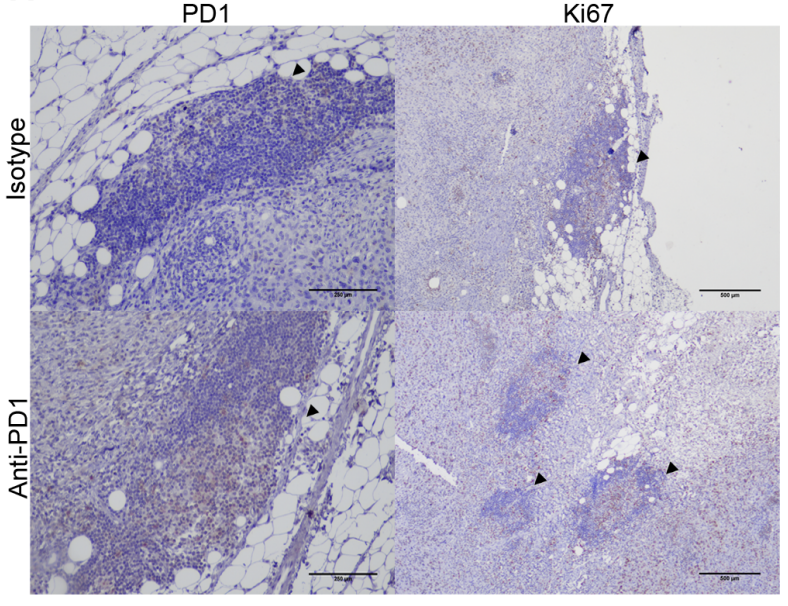

B

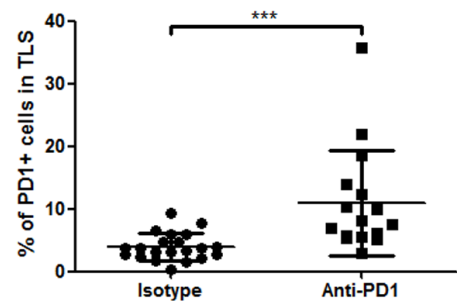

C

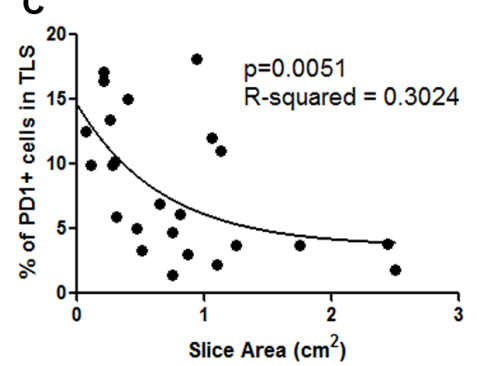

$p>0.05$

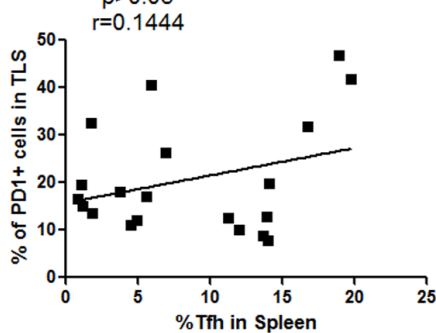

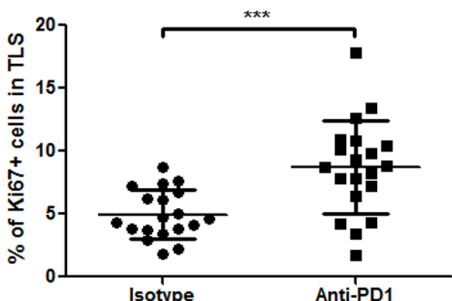
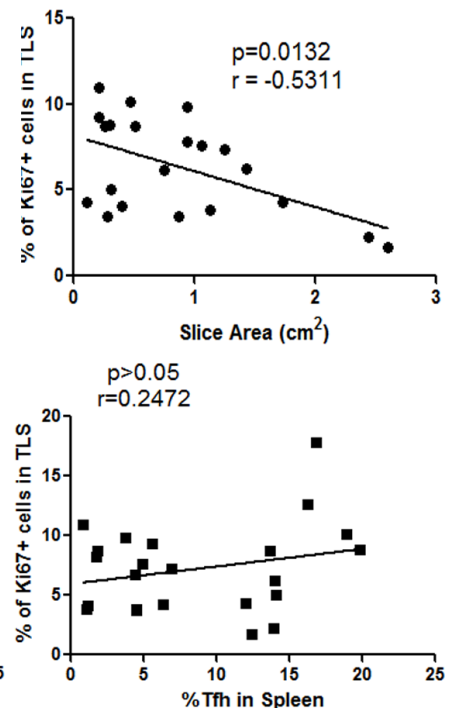
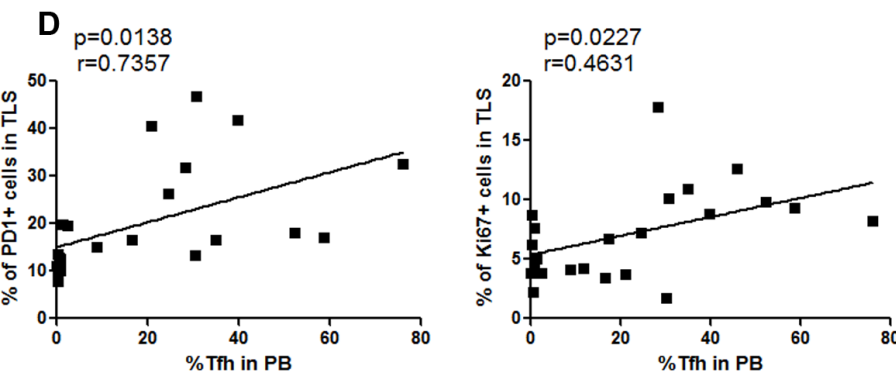

Figure 6 Antiprogrammed cell death protein 1 (anti-PD-1) treatment promotes tertiary lymphoid structures (TLS) activation. (A) Immunohistochemical images of tumor sections from anti-PD-1-treated and isotype-treated mice stained for PD-1 (left panels) ( $n=21$ and $n=16$, respectively) and Ki67 (right panels) ( $n=19$ and $n=20$, respectively). Arrowheads indicate positive staining for PD-1 and Ki67, respectively. Scale bars represent $250 \mu \mathrm{m}$ and $500 \mu \mathrm{m}$ in left and right images, respectively. (B) Percentage of PD-1+ and Ki67+ cells in TLS from tumors of isotype-treated and anti-PD-1-treated mice. (C) Correlation between tumor size and percentage of either PD-1+ (left) ( $n=24)$ or Ki67+ (right) $(n=21)$ cells in TLS. R-squared as a goodness-of-fit measure or Pearson's $r$ are shown. (D) From left to right, correlation between percentage of circulating $T$ follicular helper cells (cTfh) and either PD-1+ $(n=22)$ or Ki67+ $(n=24)$ cells in TLS, and between percentage of spleen follicular helper T cells (Tfh) and either PD$1+(n=20)$ or Ki67+ $(n=23)$ cells in TLS. Spearman's coefficient (first and third panels) or Pearson's $r$ (second and fourth panels) are shown for each correlation. ${ }^{* *} \mathrm{P}<0.001$.

tumors. These cells impair CD8+ effector function in a PD-1/PD-L1-dependent fashion and favor tumor evasion mechanisms. ${ }^{32}$ Further administration of anti-PD-1 leads to a decrease in $\mathrm{CD} 4+\mathrm{PD}^{-1}{ }^{\mathrm{hi}}$ population and restores CD8+ antitumor activity. However, anti-CTLA4-induced CD4+ PD- ${ }^{\text {hi }}$ cells show comparable B helper activity to that of their CD4+ PD-1- counterparts. Accordingly, antitumor activity of anti-CTLA-4 does not seem to rely on B cell activation, since tumors developed in SAP knockout mice are responsive to anti-CTLA-4 therapy. ${ }^{32}$

Recent reports posit a role for PD-1 as tumor suppressor in lung cancer immunodeficient models, and provide evidence that anti-PD-1 antibodies may promote both in vitro tumor cell proliferation and in vivo tumor growth in PD-1-expressing lung cancers. ${ }^{33}{ }^{34}$ However, these models are void of immune compartment, and the overall impact of combined tumor and immune PD-1 blockade has not been evaluated. In this regard, anti-PD-1-mediated tumor regression in PD-1-positive tumors could be directly related to immune tumor infiltration and anti-PD-1-mediated T cell activation potential. Hence, effective T cell activation would counteract enhanced tumor progression potentially associated with this treatment. We propose that antiPD-1-mediated increase in cTfh population and humoral response could collaborate in antineoplastic response secondary to immune system activation in this setting. Nevertheless, a comprehensive analysis of both tumor PD-1 and immune infiltration degree in patient samples is required to accurately establish their influence in antiPD-1 therapy outcome. ${ }^{35}$ In this regard, tumor PD-L1 expression in NSCLC biopsies is considered as a predictive parameter of response, and its evaluation is routinely performed to establish the indication of PD-1/PD-L1 inhibitors. However, clinical trials aimed at evaluating the predictive value of PD-L1 show contradictory results. ${ }^{36}$ In addition, assessing PD-L1 expression in tumor biopsies involves an invasive procedure with additional risk. On the contrary, the implementation of cTfh immunophenotyping by flow cytometry in peripheral blood would be an affordable minimal invasive test that could provide valuable information about potential response to anti-PD-1 treatment in these patients. 

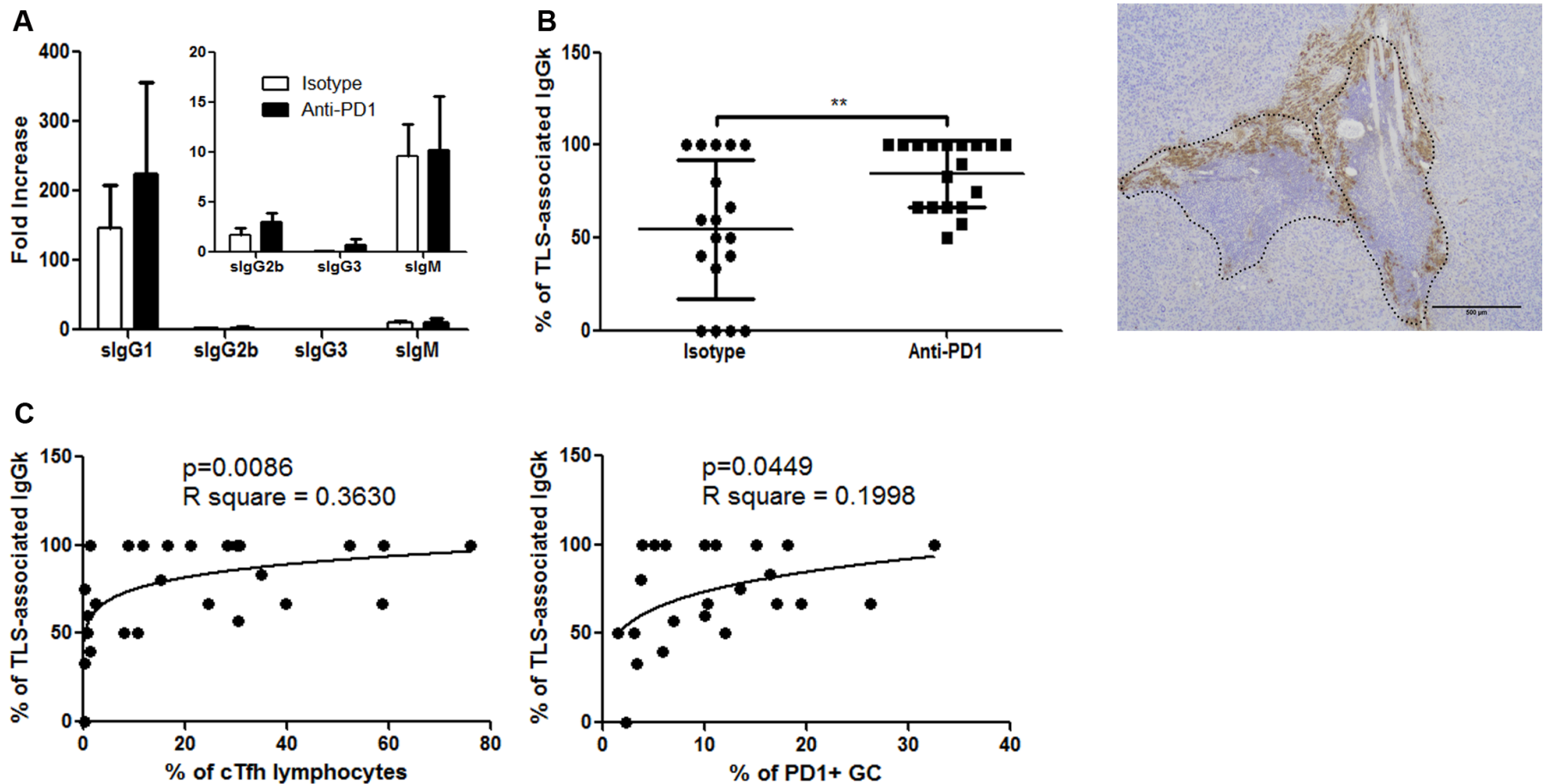

Figure 7 Antiprogrammed cell death protein 1 (anti-PD-1) therapy promotes in situ antibody generation in tertiary lymphoid structures (TLS). (A) Relative expression of secreted IgG1, IgG2b, IgG3 and IgM isotypes in whole tumor sections of anti-PD-1treated and isotype-treated mice $(n=13)$. Ig expression was normalized between tumor samples using membrane-bound lgM. (B) Left, percentage of TLS positive for lgG kappa (lgGk) staining in tumor sections from anti-PD-1-treated and isotype-treated mice $(n=18)$. Right, immunohistochemical image of tumor section stained for IgGk. Dashed lines indicate TLS boundaries. Scale bars represent $500 \mu \mathrm{m}$. (C) Correlation between percentage of IgGk-positive TLS and either percentage of circulating T follicular helper cells (cTfh) (left panel) $(n=26)$ or PD-1+ germinal centers (GC) in TLS (right panel) ( $n=23)$ in anti-PD-1-treated mice. Rsquared is shown as a goodness-of-fit measure. ${ }^{\star *} \mathrm{P}<0.01$.

In addition to Tfh expansion, PD-1 deficiency leads to an increase in Tfr cells with enhanced suppressor ability especially in peripheral blood, which suggests a role for PD-1 in Tfr generation together with PD-1-dependent limitation of Tfr release from SLOs. ${ }^{38}$ In contrast, we found a significant decrease in the proportion of circulating Tfr on anti-PD-1 treatment (figure 1F). This finding indicates that anti-PD-1 primarily affects cTfh in our model, and further supports a role for this molecule at different stages of Tfh development.

Parallel to tumor progression, we observe a systemic humoral response evidenced both by the increment in serum IgM (figure 3 ) and by the presence of active GCs in SLOs, further enhanced by anti-PD-1 treatment (figure 4 and online supplemental figure 2B). This humoral response includes circulating antitumor antibodies that can be detected in both isotype-treated and anti-PD-1treated mice (figure 3). Accordingly, the increase in active GC number promoted by anti-PD-1 does not correlate with response to therapy (figure $4 \mathrm{E}$ ). On the other hand, antiPD-1 treatment upregulates TLS number, which is indeed associated with a positive response to therapy (figure 5A). Moreover, cTfh increase secondary to anti-PD-1 treatment correlates with augmented local Ig production supported by TLS (figure 7B,C). Thus, despite TLS might generate antibodies that go out into peripheral blood and be detected in serum, ${ }^{39}$ we hypothesize that cTfh-associated local Ig production in TLS is a main effector of antitumor humoral response induced by anti-PD-1.

Humoral response triggered by tumor growth also involves the appearance of autoantibodies (online supplemental figure 1), which strongly suggests the presence of a paraneoplastic process. Paraneoplastic syndromes appear in up to $10 \%$ of patients with lung cancer. ${ }^{40}$ In squamous carcinoma they are predominantly related to hypercalcemia and to the development of autoimmune conditions. ${ }^{40}{ }^{41}$ Many of these syndromes preferentially involve cytotoxic $\mathrm{T}$ cell activation that secondarily leads to the generation of non-pathogenic autoantibodies, which are not always detectable. ${ }^{42}$ In most patients, paraneoplastic manifestations either improve or disappear on tumor treatment. ${ }^{41}$ On the other hand, anti-PD-1 leads to autoimmune-related side effects in a reduced number of cases. ${ }^{43}$ In our model, anti-PD-1 therapy slightly reduces autoantibody titer, thus reflecting a possible improvement of autoimmune condition, accompanied or not by the development of immune-related toxicity with predominant $\mathrm{T}$ cell involvement.

The presence of TLS associates with a favorable prognostic in several malignancies, including lung cancer. ${ }^{44}$ In our NSCLC model, TLS increase induced by antiPD-1 inversely correlates with tumor size (figure 5B), which is in accordance with data previously obtained in patients. ${ }^{44}$ TLS generation in solid tumors is regulated by 
mediators similar to those required for GC formation in SLOs. Thus, a specific 12-gene signature was described able to predict TLS presence within the tumor, ${ }^{45}$ which comprises an array of chemokines involved in different steps of TLS formation. Among these chemokines, CCL21 mediates homing of DC and CCR7+ naïve T cells, which contribute to generate ectopic lymphoid structures in both CCL21 transgenic mice and chronically inflamed tissues. ${ }^{46} 47$ The major role played by CCL21 in antitumor immune responses is suggested by the increase in tumor infiltrating CD8+ lymphocytes and the induction of systemic antitumor response on intratumor injection of CCL21 gene-modified DC in patients with lung cancer. ${ }^{48}$ Remarkably, our data show a specific increase in tumor CCL21 expression when treated with anti-PD-1 (figure 5B). This chemokine could participate in cTfh recruitment to anti-PD-1induced TLS, where these Tfh with increased B activation capacity would promote local antibody generation and collaborate in tumor response to therapy. Both stromal fibroblasts and cells present in high endothelial venules (HEV) have been proposed as main sources of CCL21 in TLS. ${ }^{49}$ We neither analyzed the cell type(s) responsible for nor the mechanism involved in CCL21 production in response to anti-PD-1, so further research is required to understand these issues. In addition to CCL21 increase, we detected a significant reduction in the expression of other chemokines involved in TLS formation. However, some of them have been also associated with tumor progression. For instance, CXCL13 is induced by carcinogenic agents, ${ }^{50}$ and is implicated in the development of some neoplasms as lung cancer. ${ }^{51}$ Likewise, CXCL13 expression in tumors is related with poor survival outcome in different malignancies, ${ }^{52} 53$ thus the observed CXCL13 decrease induced by antiPD-1 (online supplemental figure 3B) could contribute to control tumor progression. However, CXCL13 expression is linked to a favorable outcome in melanoma, soft tissue sarcoma and colorectal cancer. ${ }^{30} 3154$ Hence, it would be of great interest to evaluate the precise role of CXCL13 in tumor development in our model, and the actual effect of anti-PD-1 therapy on CXCL13 expression in human NSCLC. Similarly, CXCL12 is known to exert protumorigenic and prometastatic actions in NSCLC, ${ }^{55}$ which could be partially counteracted by the decreased production of this chemokine on anti-PD-1 treatment (online supplemental figure 3B). Finally, CD103+ DC-derived CXCL9 has been proposed to mediate response to anti-PD-1 therapy in a murine model of colon cancer by facilitating DC-T cell interactions. ${ }^{56}$ In this article, the authors detected an early increase in CXCL9 expression only 24 hours after starting anti-PD-1 treatment; however, we did not find changes in this chemokine at later stages (online supplemental figure 3B). These data suggest that CXCL9 could participate at initial phases of response to anti-PD-1 treatment, not related with cTfh increase.

\section{CONCLUSIONS}

We provide evidence for an anti-PD-1-induced cTfh population with increased $\mathrm{B}$ activation capacity, which participates in tumor response to anti-PD-1 therapy in NSCLC. Likewise, we show an increase in the TLS number in anti-PD-1-treated tumors, which correlates with reduced tumor growth. These structures support cTfh-associated local antibody production, which is enhanced by antiPD-1 treatment and may participate in host immune response against NSCLC tumors. These findings suggest a novel role of PD-1 in antineoplastic immune response, and support the existence of new mechanisms of action for anti-PD-1 therapy.

Acknowledgements The authors would like to thank Drs M Gómez and L Baron for editing of the manuscript, and M Vitón and E Prieto for excellent technical assistance. The authors would also like to thank Drs S Martínez-Martínez and JM Redondo-Moya for critical reading of the manuscript, and Dr F Sánchez-Madrid for help with in vivo experiments.

Contributors AA, ARR, JMS-T and RC contributed to the design of the work, the analysis and interpretation of data, and either wrote or substantively revised the manuscript. SS-A contributed to acquisition and analysis of the data and wrote the manuscript. GS-J, MA, TH and MIM contributed to acquisition of the data.

Funding This work was supported by grants to AA: FIS PI15/01491 and CIBER CARDIOVASCULAR from the Instituto de Salud Carlos III (Fondo de Investigación Sanitaria del Instituto de Salud Carlos III with co-funding from the Fondo Europeo de Desarrollo Regional; FEDER).

Competing interests None declared.

Patient consent for publication Not required.

Ethics approval All animal studies were conducted at Centro Nacional de Investigaciones Cardiovasculares (CNIC) in accordance with EU Directive 2010/63/ EU and Recommendation 2007/526/EC regarding the protection of animals used for experimental purposes.

Provenance and peer review Not commissioned; externally peer reviewed. Data availability statement Data are available on reasonable request.

Open access This is an open access article distributed in accordance with the Creative Commons Attribution Non Commercial (CC BY-NC 4.0) license, which permits others to distribute, remix, adapt, build upon this work non-commercially, and license their derivative works on different terms, provided the original work is properly cited, appropriate credit is given, any changes made indicated, and the use is non-commercial. See http://creativecommons.org/licenses/by-nc/4.0/.

\section{ORCID iD}

Arantzazu Alfranca http://orcid.org/0000-0002-3732-5816

\section{REFERENCES}

1 Siegel RL, Miller KD, Jemal A. Cancer statistics, 2019. CA A Cancer J Clin 2019;69:7-34.

2 Mellman I, Coukos G, Dranoff G. Cancer immunotherapy comes of age. Nature 2011;480:480-9.

3 Vinuesa CG, Linterman MA, Yu D, et al. Follicular helper T cells. Annu Rev Immunol 2016;34:335-68.

$4 \mathrm{He}$ J, Tsai LM, Leong YA, et al. Circulating precursor CCR7(lo) PD-1(hi) CXCR5 ${ }^{+} \mathrm{CD}^{+} \mathrm{T}$ cells indicate Tfh cell activity and promote antibody responses upon antigen reexposure. Immunity 2013;39:770-81.

5 Locci M, Havenar-Daughton C, Landais E, et al. Human circulating PD-1+CXCR3-CXCR5+ memory Tfh cells are highly functional and correlate with broadly neutralizing HIV antibody responses. Immunity 2013;39:758-69.

6 Schmitt N, Ueno H. Blood Tfh cells come with colors. Immunity 2013;39:629-30.

7 Drayton DL, Liao S, Mounzer RH, et al. Lymphoid organ development: from ontogeny to neogenesis. Nat Immunol 2006;7:344-53. 
8 Lohr M, Edlund K, Botling J, et al. The prognostic relevance of tumour-infiltrating plasma cells and immunoglobulin kappa $\mathrm{C}$ indicates an important role of the humoral immune response in nonsmall cell lung cancer. Cancer Lett 2013;333:222-8.

9 Engelhard VH, Rodriguez AB, Mauldin IS, et al. Immune cell infiltration and tertiary lymphoid structures as determinants of antitumor immunity. J Immunol 2018;200:432-42.

10 Germain C, Gnjatic S, Dieu-Nosjean M-C. Tertiary lymphoid structure-associated B cells are key players in anti-tumor immunity. Front Immunol 2015;6:67.

11 Sautès-Fridman C, Petitprez F, Calderaro J, et al. Tertiary lymphoid structures in the era of cancer immunotherapy. Nat Rev Cancer 2019;19:307-25.

12 Nguyen KB, Spranger S. Modulation of the immune microenvironment by tumor-intrinsic oncogenic signaling. J Cell Biol 2020;219. doi:10.1083/jcb.201908224. [Epub ahead of print: 06 Jan 2020].

13 Sharpe AH, Pauken KE. The diverse functions of the PD1 inhibitory pathway. Nat Rev Immunol 2018;18:153-67.

14 Nguyen LT, Ohashi PS. Clinical blockade of PD1 and LAG3--potential mechanisms of action. Nat Rev Immunol 2015;15:45-56.

15 Shi J, Hou S, Fang Q, et al. Pd-1 controls follicular T helper cell positioning and function. Immunity 2018:49:264-74.

16 Reck M, Rodríguez-Abreu D, Robinson AG, et al. Pembrolizumab versus chemotherapy for PD-L1-positive non-small-cell lung cancer. N Engl J Med 2016;375:1823-33.

17 Paz-Ares L, Luft A, Vicente D, et al. Pembrolizumab plus chemotherapy for squamous non-small-cell lung cancer. N Engl J Med 2018;379:2040-51.

18 Borghaei H, Paz-Ares L, Horn L, et al. Nivolumab versus docetaxel in advanced Nonsquamous non-small-cell lung cancer. N Engl J Med 2015;373:1627-39.

19 Gambera S, Abarrategi A, González-Camacho F, et al. Clonal dynamics in osteosarcoma defined by RGB marking. Nat Commun 2018;9:3994.

20 Martins F, Sofiya L, Sykiotis GP, et al. Adverse effects of immunecheckpoint inhibitors: epidemiology. management and surveillance 2019;16:563-80.

21 Mueller CG, Nayar S, Campos J, et al. Molecular and cellular requirements for the assembly of tertiary lymphoid structures. Adv Exp Med Biol 2018;1060:55-72.

22 Kaneko T, LePage GA, Shnitka TK. KLN205--a murine lung carcinoma cell line. In Vitro 1980;16:884-92.

23 Osmani L, Askin F, Gabrielson E, et al. Current who guidelines and the critical role of immunohistochemical markers in the subclassification of non-small cell lung carcinoma (NSCLC): moving from targeted therapy to immunotherapy. Semin Cancer Biol 2018:52:103-9.

24 Kawamoto S, Tran TH, Maruya M, et al. The inhibitory receptor PD-1 regulates IgA selection and bacterial composition in the gut. Science 2012;336:485-9.

25 Liu T, Cheng X, Ding Y, et al. Pd-1 deficiency promotes Tfh cells expansion in ITV-immunized mice by upregulating cytokines secretion. Parasit Vectors 2018;11:397.

26 Herati RS, Muselman A, Vella L, et al. Successive annual influenza vaccination induces a recurrent oligoclonotypic memory response in circulating T follicular helper cells. Sci Immunol 2017;2. doi:10.1126/ sciimmunol.aag2152. [Epub ahead of print: 17 Feb 2017].

27 Schmitt N, Bentebibel S-E, Ueno H. Phenotype and functions of memory Tfh cells in human blood. Trends Immunol 2014;35:436-42.

28 Hollern DP, Xu N, Thennavan A, et al. B cells and T follicular helper cells mediate response to checkpoint inhibitors in high mutation burden mouse models of breast cancer. Cell 2019;179:e21:1191-206.

29 Cabrita R, Lauss M, Sanna A, et al. Tertiary lymphoid structures improve immunotherapy and survival in melanoma. Nature 2020;577:561-5.

30 Helmink BA, Reddy SM, Gao J, et al. B cells and tertiary lymphoid structures promote immunotherapy response. Nature 2020;577:549-55.

31 Petitprez F, de Reyniès A, Keung EZ, et al. B cells are associated with survival and immunotherapy response in sarcoma. Nature 2020;577:556-60.

32 Zappasodi R, Budhu S, Hellmann MD, et al. Non-Conventional inhibitory CD4+Foxp3-PD-1hi T cells as a biomarker of immune checkpoint blockade activity. Cancer Cell 2018;33:1017-32.
33 Wang X, Yang X. Tumor cell-intrinsic PD-1 receptor is a tumor suppressor and mediates resistance to PD-1 blockade therapy 2020;117:6640-50.

34 Du S, McCall N, Park K, et al. Blockade of Tumor-Expressed PD-1 promotes lung cancer growth. Oncoimmunology 2018;7:e1408747.

$35 \mathrm{Yao} \mathrm{H}$, Wang H, Li C, et al. Cancer cell-intrinsic PD-1 and implications in combinatorial immunotherapy. Front Immunol 2018;9:9.

36 Hanna NH, Schneider BJ, Temin S, et al. Therapy for stage IV nonsmall-cell lung cancer without driver alterations: ASCO and $\mathrm{OH}$ (CCO) joint guideline update. J Clin Oncol 2020;38:1608-32.

37 Kluger HM, Zito CR, Turcu G, et al. Pd-L1 studies across tumor types, its differential expression and predictive value in patients treated with immune checkpoint inhibitors. Clin Cancer Res 2017:23:4270-9.

38 Sage PT, Francisco LM, Carman CV, et al. The receptor PD-1 controls follicular regulatory $T$ cells in the lymph nodes and blood. Nat Immunol 2013;14:152-61.

39 Germain C, Gnjatic S, Tamzalit F, et al. Presence of B cells in tertiary lymphoid structures is associated with a protective immunity in patients with lung cancer. Am J Respir Crit Care Med 2014;189:832-44.

40 Kanaji N, Watanabe N, Kita N, et al. Paraneoplastic syndromes associated with lung cancer. World J Clin Oncol 2014;5:197-223.

41 Holbrechts S, Gorham J, Sideris S, et al. Autoimmune paraneoplastic syndromes associated to lung cancer: a systematic review of the literature: Part 2: hematologic, cutaneous and vascular syndromes. Lung Cancer 2017;106:93-101.

42 Graus F, Dalmau J. Paraneoplastic neurological syndromes in the era of immune-checkpoint inhibitors. Nat Rev Clin Oncol 2019;16:535-48

43 El Osta B, Hu F, Sadek R, et al. Not all immune-checkpoint inhibitors are created equal: meta-analysis and systematic review of immunerelated adverse events in cancer trials. Crit Rev Oncol Hematol 2017; 119:1-12.

44 Dieu-Nosjean M-C, Goc J, Giraldo NA, et al. Tertiary lymphoid structures in cancer and beyond. Trends Immunol 2014;35:571-80.

45 Zhu G, Falahat R, Wang K, et al. Tumor-Associated tertiary lymphoid structures: gene-expression profiling and their bioengineering. Front Immunol 2017;8:767.

46 Fan L, Reilly CR, Luo Y, et al. Cutting edge: ectopic expression of the chemokine TCA4/SLC is sufficient to trigger lymphoid neogenesis. $J$ Immunol 2000;164:3955-9.

47 Barone F, Bombardieri M, Manzo A, et al. Association of CXCL13 and CCL21 expression with the progressive organization of lymphoid-like structures in Sjögren's syndrome. Arthritis Rheum 2005;52:1773-84.

48 Lee JM, Lee M-H, Garon E, et al. Phase I Trial of Intratumoral Injection of CCL21 Gene-Modified Dendritic Cells in Lung Cancer Elicits Tumor-Specific Immune Responses and $\mathrm{CD} 8^{+} \mathrm{T}$-cell Infiltration. Clin Cancer Res 2017;23:4556-68.

49 Barone F, Gardner DH, Nayar S, et al. Stromal fibroblasts in tertiary lymphoid structures: a novel target in chronic inflammation. Front Immunol 2016;7:477.

50 Wang G-Z, Cheng X, Zhou B, et al. The chemokine CXCL13 in lung cancers associated with environmental polycyclic aromatic hydrocarbons pollution. Elife 2015;4. doi:10.7554/eLife.09419. [Epub ahead of print: 13 Nov 2015].

51 Shiels MS, Pfeiffer RM, Hildesheim A, et al. Circulating inflammation markers and prospective risk for lung cancer. J Natl Cancer Inst 2013;105:1871-80.

52 Qi X-W, Xia S-H, Yin Y, et al. Expression features of CXCR5 and its ligand, CXCL13 associated with poor prognosis of advanced colorectal cancer. Eur Rev Med Pharmacol Sci 2014;18:1916-24.

$53 \mathrm{Kim}$ SJ, Ryu KJ, Hong M, et al. The serum CXCL13 level is associated with the Glasgow prognostic score in extranodal NK/Tcell lymphoma patients. J Hematol Oncol 2015;8:49.

54 Bindea G, Mlecnik B, Tosolini M, et al. Spatiotemporal dynamics of intratumoral immune cells reveal the immune landscape in human cancer. Immunity 2013;39:782-95.

55 Wald O. Cxcr4 based therapeutics for non-small cell lung cancer (NSCLC). J Clin Med 2018;7. doi:10.3390/jcm7100303. [Epub ahead of print: 25 Sep 2018].

56 Chow MT, Ozga AJ, Servis RL, et al. Intratumoral activity of the CXCR3 chemokine system is required for the efficacy of anti-PD-1 therapy. Immunity 2019;50:1498-512. 